\title{
Impact of Vibration and Agitation Speed on Dissolution of USP Prednisone Tablets RS and Various IR Tablet Formulations
}

\author{
Nicole Seeger, ${ }^{1}$ Sigrid Lange, ${ }^{1}$ and Sandra Klein ${ }^{1,2}$
}

Received 31 December 2014; accepted 11 June 2015; published online 24 June 2015

\begin{abstract}
Dissolution testing is an in vitro procedure which is widely used in quality control (QC) of solid oral dosage forms and, given that real biorelevant test conditions are applied, can also be used as a predictive tool for the in vivo performance of such formulations. However, if a dissolution method is intended to be used for such purposes, it has to deliver results that are only determined by the quality of the test product, but not by other variables. In the recent past, more and more questions were arising on how to address the effects of vibration on dissolution test results. The present study was performed to screen for the correlation of prednisone dissolution of USP Prednisone Tablets RS with vibration caused by a commercially available vibration source as well as to investigate how drug release from a range of immediate release formulations containing class 1-4 drugs of the biopharmaceutical classification scheme is affected by vibration when performing dissolution experiments at different agitation rates. Results of the present study show that the dissolution process of oral drug formulations can be affected by vibration. However, it also becomes clear that the degree of which a certain level of vibration impacts dissolution is strongly dependent on several factors such as drug properties, formulation parameters, and the design of the dissolution method. To ensure the establishment of robust and predictive dissolution test methods, the impact of variation should thus be considered in method design and validation.
\end{abstract}

KEY WORDS: dissolution; USP prednisone calibrator tablets; variability; vibration meter; vibration source.

\section{INTRODUCTION}

Dissolution testing is an in vitro procedure which is widely used in quality control (QC) of solid oral dosage forms and, given that real biorelevant test conditions are applied, can also be used as a predictive tool for the in vivo performance of such formulations. However, if a dissolution method is intended to be used for such purposes, it has to be robust, i.e., it has to deliver stable and reliable results that are only determined by the quality of the test product, but not by other variables such as for instance the condition of the dissolution apparatus, the lab environment, the operator of the instrument, or the day of the experiment. To ensure that the results of a dissolution experiment solely reflect the variability in product quality but not that of the test conditions, specific calibration procedures for dissolution apparatus have been established over the last decades. Nowadays, these procedures are receiving a great deal of attention [1]. Whereas there is a consensus in

\footnotetext{
${ }^{1}$ Department of Pharmacy, Institute of Biopharmaceutics and Pharmaceutical Technology, Center of Drug Absorption and Transport, Ernst Moritz Arndt University, 3 Felix Hausdorff Street, Greifswald, 17487, Germany.

${ }^{2}$ To whom correspondence should be addressed. (e-mail: sandra.klein@uni-greifswald.de)
}

the need of a mechanical calibration procedure to confirm that the physical conditions of the test equipment are in agreement with the specifications made in the USP, there is an ongoing discussion on how to assess the impact of additional operational parameters such as degassing of the dissolution medium, dosage form placement in the dissolution vessel, use of different basket clips or sinker types, and incidental vibration [1]. The United States Pharmacopoeial Convention has established the performance verification test (PVT) to assess the assembly, analyst performance, and analytical procedures belonging to a dissolution experiment [2]. However, it is still not clear whether a simple experiment with formerly two and nowadays just one immediate release (IR) tablet formulation type(s) is appropriate to decide on the applicability of a dissolution test system in QC and/or formulation development. In addition to this, in the recent past, more and more questions were arising on how to address the effects of vibration on dissolution test results [3-7]. Vibration can result in the addition of energy to a system, e.g., a dissolution bath and can thus potentially alter the results of an experiment. In the USP therefore, the statement "No part of the assembly, including the environment in which the assembly is placed, contributes significant motion, agitation, or vibration beyond that due to the smoothly rotating stirring element" [5] can be found, but from this simple statement, it is not clear, what are critical 
vibration levels and how to assess them in calibration of the equipment and in routine dissolution testing.

As early as in the early 1970s, Beyer and Smith [8] had performed standardized studies in evaluating the impact of internal and external vibration on dissolution rates of tablets in the rotating basket system (USP apparatus 1) and also to check what was the principal source of vibration in these particular studies. It became clear that vibration can have a significant impact on dissolution results and that the effect of vibration is much more pronounced at low agitational rates. In the cited manuscript, data for just one tablet formulation (tolbutamide tablets USP) are presented. However, based on their observations, the authors conclude that when, e.g., plotting the time required for $50 \%$ of drug released/dissolved from a formulation versus the agitational rate in revolutions per minute (rpm) applied in the corresponding experiment, one will obtain plots that vary considerably from product to product and, to a lesser extent, from lot to lot of the same product. Therefore to their opinion, control of vibration might be more important for some products than for others, but definitely must be addressed when establishing dissolution specifications. Beyer and Smith further suggest two potential courses of action for this purpose which include (a) carefully controlling the vibration within a specified limit as defined by an objective test procedure or (b) reducing the vibration effects by selecting a sufficient agitation speed for the experiment.

In the following decades, several more case studies on the impact of vibration on dissolution test results were published. Embil and Torosian had studied vibration effects on drug release of enteric-coated aspirin tablets in the paddle apparatus [9]. Similar to Beyer and Smith, they observed a more pronounced vibration effect resulting in a decrease in dissolution times at low paddle speeds $(50 \mathrm{rpm})$ which could be overcome by increasing the paddle speed to $200 \mathrm{rpm}$. In a collaborative study, in which seven laboratories participated, Kaniwa et al. tried to develop a dissolution standard for evaluating critical vibration levels in the rotating basket and the paddle apparatus run at $50 \mathrm{rpm}$ [3]. Even though their approach was different from that applied in previous studies, they made the same observations, namely that at this low agitation speeds, a certain level of vibration can have a significant effect on the dissolution results.

Today, this means more than 30 years after the publication of results from the Beyer and Smith study, the discussion on how to assess vibration and how to overcome the problems that come along with internal and external vibration in a dissolution experiment is still ongoing. In the recent past, various activities were started (a) to better understand vibration, (b) to determine how and where to best measure vibration in a dissolution setup, and (c) to establish meaningful vibration limits for dissolution testing.

Due to the inability to properly measure vibration levels at the most relevant point of a dissolution apparatus which would be inside a dissolution vessel during operation, in recent studies, accelerometers/vibration meters were used to measure environmental and induced vibration at various locations of a dissolution instrument in the course of dissolution experiments with USP Prednisone Tablets RS [4, 5, 10-12]. In all of the cited studies, it became obvious that one of the biggest challenges is to determine the right location for recording vibration levels that are relevant to the dissolution process in the vessel. Moreover, results of the different studies are in good agreement since independent on the apparatus type used in the study, in all of the cited studies, the most pronounced vibration effect was seen in the prednisone experiments performed at $50 \mathrm{rpm}$. The latter observation is not surprising, since the USP Prednisone Tablets RS had been developed to detect operational sources of error that cannot be checked/adjusted in a mechanical calibration procedure. However, as stated before, beside vibration, these operational sources of error include also improper media degassing, vessels from different sources, irregularities on the inner surface of glass vessels, an inadequate or improper sampling procedure, etc. All these sources can impact both the amount of prednisone released and the variability observed in dissolved prednisone values [2]. Thus, the presence of significant vibration will become visible in the results of a PVT experiment. However, because of the multitude of parameters that can affect results of the PVT experiment, a non-conforming PVT result cannot necessarily be correlated with a particular source of error or a specific vibration level. Therefore, there is an ongoing discussion on how to measure vibration during a dissolution experiment and how to set acceptable vibration levels that constitute a suitable testing environment $[5,11]$.

The present study was performed to screen for the correlation of prednisone dissolution of USP Prednisone Tablets RS with vibration caused by a commercially available vibration source as well as to investigate how drug release from a range of immediate release (IR) formulations containing class $1-4$ drugs of the biopharmaceutical classification scheme (BCS) is affected by vibration when performing dissolution experiments at different agitation rates.

\section{MATERIALS AND METHODS}

\section{Materials}

The first set of experiments was performed with USP Prednisone Tablets RS $10 \mathrm{mg}$ lot \# P1I300. For the second set of experiments, IR tablets containing drugs that belong to different BCS classes [13], i.e., metoprolol (BCS 1), indometacin (BCS 2), atenolol (BCS 3), and furosemide (BCS 4) [14] were selected as model formulations. The following marketed formulations were used:

- Meprolol $^{\text {TM }} 100$ mg (metoprolol tartrate), batch \# 2000164, TAD Pharma, Cuxhaven, Germany

- Indometacin AL 50 mg, batch \# 53115, ALIUD® PHAR MA, Laichingen, Germany

- JuventalTM 100 mg, batch \# 909120, Henning Arzneimittel GmbH \& Co KG, Floersheim, Germany

- Furosemid AL 40 mg, batch \# 02673, ALIUD® PHARMA, Laichingen, Germany

USP Prednisone Tablets RS $10 \mathrm{mg}$ lot \# P1I300 and USP Prednisone Reference Standard lot \# O0G356 were purchased from USP Rockville, MD, USA. The different IR tablet formulations were obtained from a public pharmacy by prescription. All other reference standards and compounds were of analytical grade and purchased commercially. 


\section{Apparative Setup}

\section{Dissolution System}

All experiments were performed with an USP Apparatus 2 (DT 600 HH, ERWEKA, Heusenstamm, Germany).

\section{Vibration System}

The vibration source was a ButtkickerTM Gamer (The Guitammer Company, Westerville, OH, USA) attached to the dissolution bath (Fig. 1). Three single-axis accelerometers Model 2012-002 (Silicon Designs, Inc. Issaquah, WA, USA) were used to monitor vibration levels in three directions [12]. For this purpose, they were fastened to an aluminum cube (sensor block with a side length of $5 \mathrm{~cm}$ ) and then placed onto one of the designated spots on the vessel plate (Fig. 1) to measure the vibration along one of the three axes/directions $(\mathrm{x}, \mathrm{y}, \mathrm{z})$. Double-sided tape was used to keep the aluminum cube in place over the entire experiment. Data acquisition took place via a National Instruments (NI) DAQ USB-321 interface and using LabVIEW (version 8.6, NI) software for monitoring the output of the accelerometers.

The reason for selecting this particular vibration source and accelerometer was to be able to compare study results of the present study with those resulting from a collaborative study on the effects of vibration on dissolution test results. Since in previous studies conducted at Washington University and in the Division of Pharmaceutical Analysis (DPA) laboratories at US FDA [10-12] a strong correlation between the amplitude of induced vibration and dissolution results was obtained with an USP apparatus 2 of a certain brand, DPA, representatives of USP and five different manufacturers of dissolution equipment had agreed to perform a collaborative study on the effects of vibration on dissolution test results. The objective of this study was to establish methodology for controlled (amplitude and frequency) generation of vibration, measurement of vibration at appropriate locations on the dissolution apparatus, and correlation of the measured vibration with dissolution results obtained with a variety of models of basket and paddle dissolution apparatuses from multiple manufacturers. For performing this study, the collaborators had agreed to follow the same study protocol, i.e., using the same set of accelerometers, the same vibration source, and to follow the same test procedure, i.e., the study protocol that had been drafted by DPA (DPA Study 06R-3) [12], and the study was also intended to be performed with the USP Prednisone Calibrator Tablets. Unfortunately, since many of the manufacturers had dropped out for no significant reason, the study had never been completed. However, since our intention was to follow an official protocol and to compare results obtained with BCS class 1-4 formulations with those obtained with the USP Prednisone Calibrator Tablets, using the vibration system described in the draft study protocol was a good point to start.

\section{Experimental Setup}

\section{Mechanical Calibration and Performance Verification Test}

Before starting the vibration study, a mechanical calibration of the dissolution apparatus as well as a performance verification test (PVT) was performed according to the FDA Guidance for Industry on The Use of Mechanical Calibration of Dissolution Apparatus 1 and 2 [15] and the detailed description in the document "Mechanical Qualification of Dissolution Apparatus 1 and 2" which is available on the FDA web site (http://www.fda.gov/downloads/AboutFDA/ CentersOffices/CDER/UCM142492.pdf). The test parameters for the mechanical calibration included vessel and paddle dimensions, horizontality of the dissolution bath, shaft wobble, paddle verticality, vessel centering, vessel verticality, paddle depth, rotational speed, and temperature. A single-stage performance verification test (PVT) was performed for six positions as described in USP chapter $<711>$ and the certificate coming along with the calibrator tablets. USP Prednisone Tablets RS $10 \mathrm{mg}$ and USP Prednisone Reference Standard lot \# P1I300 were used to perform the PVT. Since the instrument was dedicated to be used as a paddle apparatus, the PVT was only performed for the paddle mode.

\section{Mapping Experiments}

Before running release experiments, with the apparatus being in its operational position, i.e., the vessels filled with $500 \mathrm{~mL}$ of deaerated water kept at $37^{\circ} \mathrm{C}$ and the paddles running at $50 \mathrm{rpm}$, the resonance frequency of the apparatus was determined by placing the aluminum cube with the attached accelerometers at positions 1, 3, 5, 7, and 9 on the vessel plate (Fig. 1) and applying a frequency range of 10-
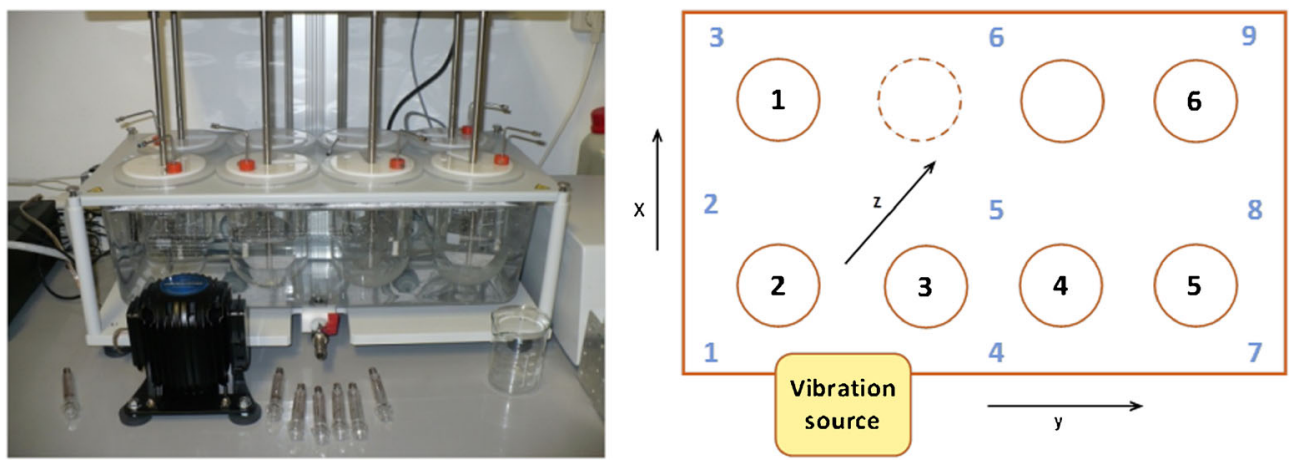

Fig. 1. Experimental setup for the vibration experiments: dissolution bath with vibration source (left), vessel plate with vessel locations and accelerometer positions (right) 
$100 \mathrm{~Hz}$ at $10 \mathrm{~Hz}$ intervals. Subsequently, vibration distribution on the apparatus vessel plate was mapped at positions 1-9 to determine the most sensitive position at which to measure vibration in the dissolution experiments. For this purpose, three different levels of vibration ranging from slight to forced vibrations applied at the resonance frequency of the apparatus (vibration levels were adjusted by increasing the vibration input by one scale division per level on the Buttkicker ${ }^{\mathrm{TM}}$ Gamer control panel) were applied and acceleration ( $g$ RMS) was recorded in $\mathrm{x}, \mathrm{y}$, and $\mathrm{z}$ directions at the most sensitive position on the vessel plate.

\section{Prednisone Experiments}

All dissolution experiments with USP Prednisone Tablets RS were performed according to the cited collaborative study protocol designed to comply with the DPA's objective to better understand vibration: Experiments were performed in $500 \mathrm{~mL}$ of deionized water at $37^{\circ} \mathrm{C}$. Before starting the vibration studies, baseline dissolution profiles were established at three paddles speeds (50/75/100 rpm) by performing dissolution tests ( $n=6$ per paddle speed) under optimum conditions (properly deaerated media, no vibration). Samples $(10 \mathrm{~mL})$ were taken manually at 10, 20, 30, 60, and 90 min using a $10 \mathrm{~mL}$ glass syringe (Fortuna ${ }^{\mathrm{TM}}$ Optima ${ }^{\mathrm{TM}}$ Luer Lock, Wertheim, Germany), and the sampling volume was replaced with fresh medium. Subsequently, experiments were performed at three different levels of vibration ranging from slight to forced vibrations applied at the resonance frequency of the apparatus (vibration levels were adjusted by increasing the vibration input by one scale division per level on the ButtkickerTM Gamer control panel). Acceleration (g RMS), displacement (mil), and velocity $(\mathrm{mm} / \mathrm{s})$ were recorded in $\mathrm{x}, \mathrm{y}$, and $\mathrm{z}$ directions at the most sensitive position on the vessel plate. Following appropriate dilution, samples were analyzed at $242 \mathrm{~nm}$ using a UV spectrophotometer (U 2000, Hitachi Ltd., Tokyo, Japan) equipped with a $10-\mathrm{mm}$ cuvette.

Finally, dissolution results were investigated for the impact of vibration on dissolution rates by correlating vibration levels, agitation rate, and the obtained dissolution profile.

\section{Experiments with BCS Class 1-4 Formulations}

All experiments with the four model formulations were performed in a media volume of $500 \mathrm{~mL}$ at $37 \pm 0.5^{\circ} \mathrm{C}$. Simulated gastric fluid without pepsin (SGFsp) USP 35 pH 1.2 was used as the test medium for metoprolol, whereas simulated intestinal fluid without pancreatin (SIFsp) USP 35 pH 6.8 was used for indometacin, atenolol, and furosemide. Special attention was given to achieve sink conditions in all experiments. Sink conditions were defined as maintaining a volume of dissolution media that is at least five times greater than the volume at the saturation point of the drug contained in the drug delivery system being tested. Baseline dissolution profiles were established at three paddle speeds (50, 75, $100 \mathrm{rpm})$ by performing dissolution tests ( $n=6$ per paddle speed) under optimum conditions (properly deaerated media, no vibration). Samples $(5 \mathrm{~mL})$ were removed at predetermined time points using a $5 \mathrm{~mL}$ glass syringe (Fortuna ${ }^{\mathrm{TM}}$ Optima ${ }^{\mathrm{TM}}$ Luer Lock, Wertheim, Germany), and the sampling volume was immediately replaced wit fresh medium. As in the prednisone experiments, in a next step, experiments were performed at three different levels of vibration (vibration levels were adjusted by increasing the vibration input by one scale division per level on the ButtkickerTM Gamer control panel) at the resonance frequency of the apparatus. Acceleration (g RMS), displacement (mil), and velocity $(\mathrm{mm} / \mathrm{s})$ were recorded in $\mathrm{x}, \mathrm{y}$, and $\mathrm{z}$ directions at the most sensitive position on the vessel plate. Following appropriate dilution, samples were analyzed at $270 \mathrm{~nm}$ (IbuHexal'TM akut 200), $273 \mathrm{~nm}$ (Meprolol 100 and Juvental $100 \mathrm{mg}$ ), and $330 \mathrm{~nm}$ (Furosemid AL $40 \mathrm{mg}$ ) using a UV spectrophotometer (U 2000, Hitachi Ltd., Tokyo, Japan) equipped with a $10-\mathrm{mm}$ cuvette. Finally, dissolution results were investigated for the impact of vibration on dissolution rates by correlating vibration levels, agitation rate, and the obtained dissolution profile.

\section{RESULTS AND DISCUSSION}

\section{Mechanical Calibration and Performance Verification Test}

Each vessel and each paddle conformed to the dimensions given in the USP General Chapter $<711>$. All other parameters were in accordance with USP requirements, and the equipment passed the mechanical calibration. Results from the PVT were as follows: The geometric mean (GM) of six vessels was $37.94 \%$ (limits, $25-41 \%$ ) of the labeled amount of prednisone dissolved in $30 \mathrm{~min}$ at $50 \mathrm{rpm}$. The percent coefficient of variation (\%CV) was 2.78 (limit: 6.8). As both GM and \%CV were in the limits for USP Prednisone Tablets RS $10 \mathrm{mg}$ lot \# P1I300, the assembly passed the PVT.

\section{Mapping Experiments}

The resonance frequency of the apparatus measured was $50 \mathrm{~Hz}$, and position 2 (Fig. 1) was determined as the most
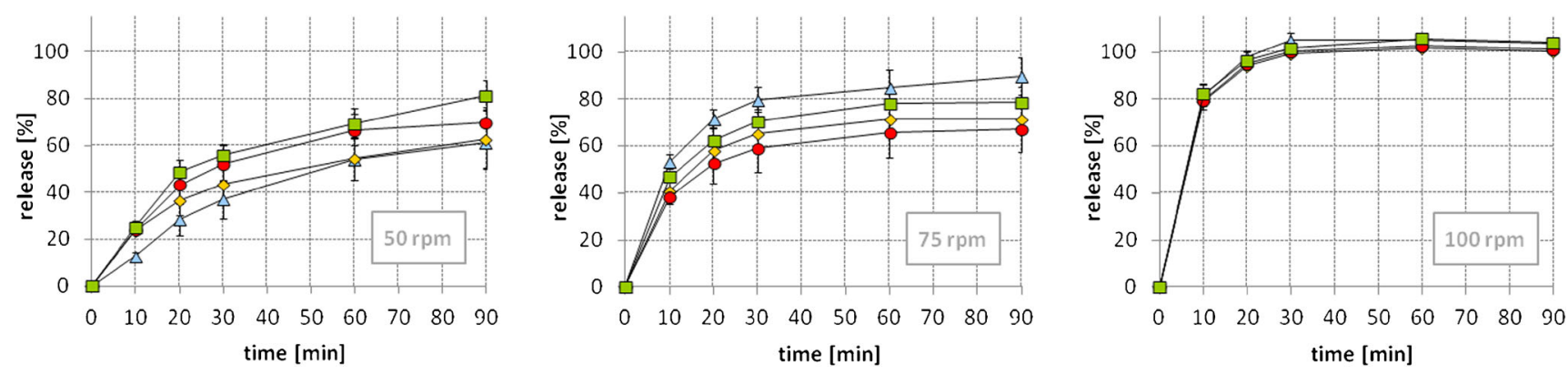

Fig. 2. Drug release (mean of $n=6 \pm \mathrm{SD}$ ) of USP Prednisone Tablets RS $10 \mathrm{mg}$ tablets at paddle speeds of 50 , 75, and $100 \mathrm{rpm}$ and different vibration levels (triangle no vibration, diamond level 1, circle level 2, and square level 3) 
Table I. Average Acceleration Values (g, RMS) Measured Over the Release Experiments with USP Prednisone Tablets RS 10 mg Tablets at Paddle Speeds of 50, 75, and $100 \mathrm{rpm}$ and Different Vibration Levels

\begin{tabular}{|c|c|c|c|c|}
\hline \multirow[b]{2}{*}{ Agitation rate } & \multirow[b]{2}{*}{ Vibration level } & \multicolumn{3}{|c|}{ Acceleration (g, RMS) } \\
\hline & & $\mathrm{z}$ & $\mathrm{y}$ & $\mathrm{x}$ \\
\hline $50 \mathrm{rpm}$ & No vibration & $0.0097 \pm 0.0021$ & $0.0018 \pm 0.0008$ & $0.0014 \pm 0.0005$ \\
\hline $50 \mathrm{rpm}$ & Level 1 & $0.0107 \pm 0.0034$ & $0.0044 \pm 0.0007$ & $0.0034 \pm 0.0005$ \\
\hline $50 \mathrm{rpm}$ & Level 2 & $0.0101 \pm 0.0013$ & $0.0068 \pm 0.0005$ & $0.0045 \pm 0.0002$ \\
\hline $50 \mathrm{rpm}$ & Level 3 & $0.0331 \pm 0.0040$ & $0.0609 \pm 0.0023$ & $0.0272 \pm 0.0009$ \\
\hline $75 \mathrm{rpm}$ & No vibration & $0.0105 \pm 0.0023$ & $0.0061 \pm 0.0004$ & $0.0036 \pm 0.0003$ \\
\hline $75 \mathrm{rpm}$ & Level 1 & $0.0173 \pm 0.0012$ & $0.0050 \pm 0.0004$ & $0.0039 \pm 0.0003$ \\
\hline $75 \mathrm{rpm}$ & Level 2 & $0.0130 \pm 0.0015$ & $0.0035 \pm 0.0006$ & $0.0034 \pm 0.0003$ \\
\hline $75 \mathrm{rpm}$ & Level 3 & $0.0686 \pm 0.0018$ & $0.0271 \pm 0.0031$ & $0.0290 \pm 0.0005$ \\
\hline $100 \mathrm{rpm}$ & No vibration & $0.0064 \pm 0.0007$ & $0.0063 \pm 0.0004$ & $0.0040 \pm 0.0002$ \\
\hline $100 \mathrm{rpm}$ & Level 1 & $0.0076 \pm 0.0025$ & $0.0057 \pm 0.0005$ & $0.0049 \pm 0.0003$ \\
\hline $100 \mathrm{rpm}$ & Level 2 & $0.0073 \pm 0.0006$ & $0.0065 \pm 0.0004$ & $0.0034 \pm 0.0003$ \\
\hline $100 \mathrm{rpm}$ & Level 3 & $0.0772 \pm 0.0032$ & $0.0195 \pm 0.0006$ & $0.0290 \pm 0.0008$ \\
\hline
\end{tabular}

sensitive position for vibration measurement. Average acceleration values ( $\mathrm{g}, \mathrm{RMS}$ ) measured in $\mathrm{x}, \mathrm{y}$, and $\mathrm{z}$ directions at this point were $0.0158,0.0030$, and 0.0031 at level $1 ; 0.0150$, 0.0026 , and 0.0028 at level 2 ; and $0.0777,0.0304$, and 0.281 at level 3, respectively. The second-most sensitive position was position 9, were average acceleration values ( $g, R M S$ ) in $x, y$, and $\mathrm{z}$ directions were $0.0062,0.0026$, and 0.0042 at level 1 ; $0.0062,0.0046$, and 0.0043 at level 2; and 0.0425, 0.0140, and 0.070 at level 3 , respectively. Based on the results of the mapping experiments, the aluminum block with the attached accelerometers was fixed at position 2 during all dissolution experiments.

\section{Prednisone Experiments}

Figure 2 shows drug release of USP Prednisone Tablets RS at paddle speeds of 50, 75, and $100 \mathrm{rpm}$ under optimal test conditions (properly degassed deionized water and no external vibration caused) and at three different levels of vibration. Tables I and II show the corresponding acceleration and displacement values measured in three different directions at position 2 of the vessel plate.

The release profiles indicate that drug release from USP Prednisone Tablets RS was strongly affected by both vibration and agitation speed. Overall, in the present setup, the agitation speed seemed to have an even more pronounced impact on prednisone dissolution than has vibration. At the official agitation speed of $50 \mathrm{rpm}$ even without any external vibration, a relatively high variability became obvious when comparing prednisone dissolution in the six different vessels. In all vessels, drug release was incomplete within the test duration of $90 \mathrm{~min}$ and the dissolution rate increased with increasing levels of vibration. At $50 \mathrm{rpm}$, also a clear coning effect could be observed. This was most pronounced in the baseline experiment where no external vibration was caused. Coning is often a source of high variation, and dissolution methods where coning can be observed have already proven to be sensitive toward vibration [11]. Results obtained at $75 \mathrm{rpm}$ were still characterized by a high variability, but interestingly, dissolution rates did not proportionally increase with the vibration level. This observation was rather unexpected and at

Table II. Average Displacement Values (mil) Measured Over the Release Experiments with USP Prednisone Tablets RS 10 mg Tablets at Paddle Speeds of 50, 75, and $100 \mathrm{rpm}$ and Different Vibration Levels

\begin{tabular}{|c|c|c|c|c|}
\hline \multirow[b]{2}{*}{ Agitation rate } & \multirow[b]{2}{*}{ Vibration level } & \multicolumn{3}{|c|}{ Displacement (mil) } \\
\hline & & $\mathrm{Z}$ & $\mathrm{y}$ & $\mathrm{x}$ \\
\hline $50 \mathrm{rpm}$ & No vibration & $0.5998 \pm 0.6002$ & $0.1246 \pm 0.1614$ & $0.0986 \pm 0.1250$ \\
\hline $50 \mathrm{rpm}$ & Level 1 & $0.7758 \pm 0.8821$ & $0.2290 \pm 0.2087$ & $0.1536 \pm 0.1731$ \\
\hline $50 \mathrm{rpm}$ & Level 2 & $0.5450 \pm 0.4248$ & $0.2204 \pm 0.0863$ & $0.1253 \pm 0.0746$ \\
\hline $50 \mathrm{rpm}$ & Level 3 & $0.7944 \pm 0.5530$ & $2.0736 \pm 0.0634$ & $0.8409 \pm 0.0407$ \\
\hline $75 \mathrm{rpm}$ & No vibration & $0.6570 \pm 0.6597$ & $0.2593 \pm 0.1387$ & $0.1487 \pm 0.1237$ \\
\hline $75 \mathrm{rpm}$ & Level 1 & $0.8211 \pm 0.4658$ & $0.1633 \pm 0.1273$ & $0.1102 \pm 0.1063$ \\
\hline $75 \mathrm{rpm}$ & Level 2 & $0.6827 \pm 0.5019$ & $0.1630 \pm 0.1312$ & $0.1149 \pm 0.1043$ \\
\hline $75 \mathrm{rpm}$ & Level 3 & $1.1931 \pm 0.4617$ & $0.9339 \pm 0.1115$ & $0.4265 \pm 0.0651$ \\
\hline $100 \mathrm{rpm}$ & No vibration & $0.2454 \pm 0.0930$ & $0.1955 \pm 0.0264$ & $0.1029 \pm 0.0180$ \\
\hline $100 \mathrm{rpm}$ & Level 1 & $0.5197 \pm 0.6283$ & $0.2022 \pm 0.1355$ & $0.1370 \pm 0.1121$ \\
\hline 100 rpm & Level 2 & $0.3314 \pm 0.1830$ & $0.1827 \pm 0.0312$ & $0.0886 \pm 0.0307$ \\
\hline $100 \mathrm{rpm}$ & Level 3 & $1.3421 \pm 0.0690$ & $0.2891 \pm 0.0175$ & $0.4862 \pm 0.0198$ \\
\hline
\end{tabular}



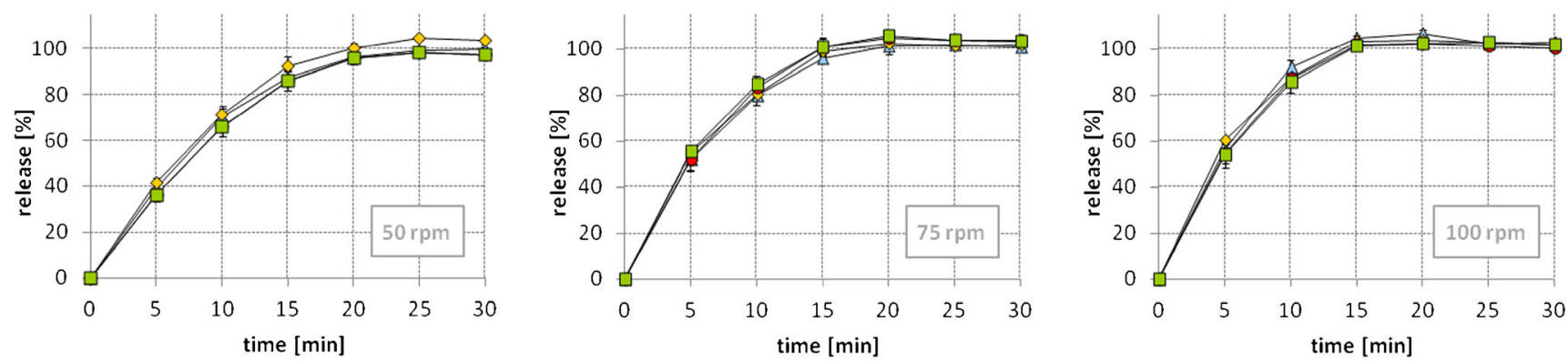

Fig. 3. Drug release (mean of $n=6 \pm \mathrm{SD}$ ) of Meprolol ${ }^{\mathrm{TM}} 100 \mathrm{mg}$ tablets at paddle speeds of 50,75 , and $100 \mathrm{rpm}$ and different vibration levels (triangle no vibration, diamond level 1, circle level 2, square level 3)

this point; there is not yet a clear explanation for the observed rank order of the dissolution profiles. However, as can be seen in Tables I-II, both acceleration and displacement did not necessarily increase when increasing the vibration input by one-scale division per level which might possibly be the reason for these observations. The dissolution profiles resulting from the experiments performed at $100 \mathrm{rpm}$ were almost superimposable, hardly affected by vibration, and drug release was complete within only $30 \mathrm{~min}$. Overall, the results from this test series confirm that under certain test conditions, particularly those applied in the official PVT, USP Prednisone Tablets RS are applicable for detecting significant vibration. However, as can be seen in the profiles obtained at different agitation speeds and with different levels of vibration applied, alone with dissolution profiles obtained in a PVT, it will most likely not be possible to predict whether another dissolution method (e.g., an official dissolution method for a certain drug product) will be sensitive to the same level of vibration or not. Based on this consideration and as a first attempt to determine meaningful/critical vibration levels for other drug products, in the next step dissolution of a set of IR formulations containing compounds from different BCS classes was studied to screen if the observations made in the prednisone experiments would also be seen from other IR tablet formulations when using the same test settings.

\section{Experiments with BCS Class 1-4 Formulations}

Dissolution profiles obtained from the BCS class 1-4 formulations indicate that the different model formulations were sensitive toward vibration to a different extent (Figs. 3, 4, 5, and 6). As in the prednisone test series, in most of the experiments, the agitation rate had a more significant impact on drug release than had vibration. This was particularly true for the experiments performed with the IR tablets containing the poorly soluble compounds indometacin (Fig. 4) and furosemide (Fig. 6). For these formulations, dissolution rate significantly increased with increasing agitation speed. Whereas at all paddle speeds, furosemide dissolution was complete within the set test duration, at a paddle speed of $50 \mathrm{rpm}$, indometacin release was much slower, and the total amount release after 30 min was almost $20 \%$ lower than at higher paddle speeds. Despite containing the highly soluble (BCS class 3) compound, atenolol also the dissolution rate of Juvental ${ }^{\mathrm{TM}}$ tablets increased with increasing agitation speeds. In contrast, Meprolol ${ }^{\mathrm{TM}}$, the IR formulation containing metoprolol showed very rapid release, i.e., $\geq 85 \%$ of the dose dissolved within $\leq 15 \mathrm{~min}$ and also no impact of vibration on drug release could be observed for this formulation.

Interestingly and as already observed in the prednisone experiments, dissolution rates often increased with increasing agitation speeds but did not necessarily proportionally increase with the vibration level, as can be seen for the dissolution of furosemide, a poorly soluble compound at $75 \mathrm{rpm}$ (Fig. 6). Also, the impact of coning (which is typically a result of a rather poor dissolution test design) on the variability of the dissolution results was confirmed as can, for instance, be seen in the dissolution results of atenolol, a highly soluble compound being formulated into a poorly disintegrating dosage form, where the excipients cause a coning effect in the experiments performed at $50 \mathrm{rpm}$ (Fig. 5).

Results of the present vibration study are in good agreement with the conclusions made by Beyer and Smith [8]. They clearly show that vibration can have a significant impact on dissolution results. In accordance with the Beyer and Smith study, the effect of vibration was often more pronounced at
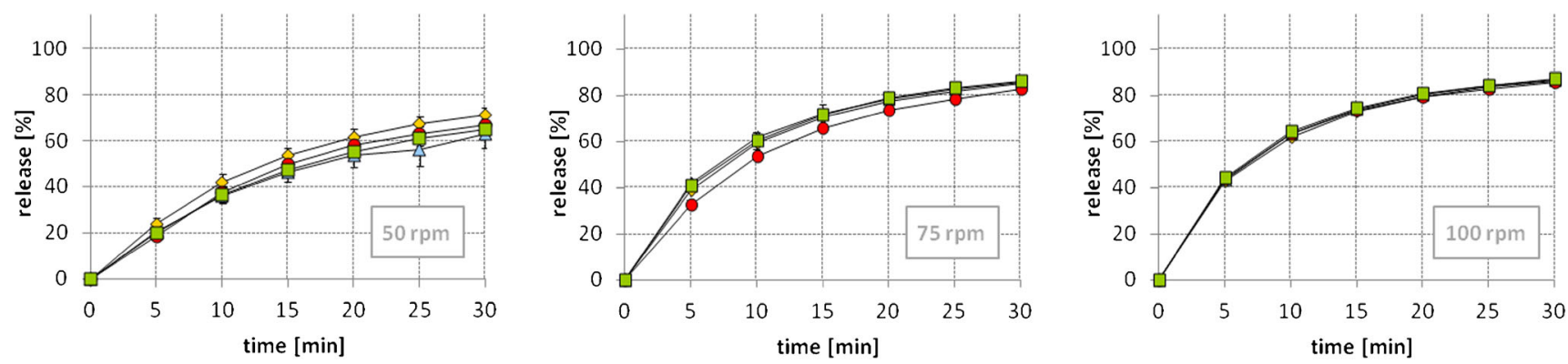

Fig. 4. Drug release (mean of $n=6 \pm \mathrm{SD}$ ) of Indometacin AL $50 \mathrm{mg}$ tablets at paddle speeds of 50, 75, and $100 \mathrm{rpm}$ and different vibration levels (triangle no vibration, diamond level 1, circle level 2, square level 3) 

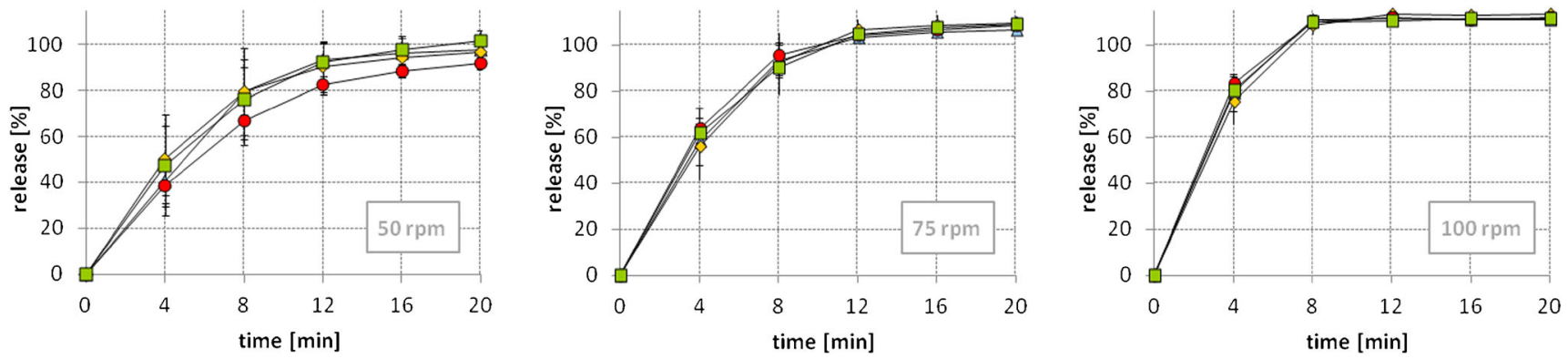

Fig. 5. Drug release (mean of $\mathrm{i}=6 \pm \mathrm{SD}$ ) of Juvental ${ }^{\mathrm{TM}} 100 \mathrm{mg}$ tablets at paddle speeds of 50,75 , and $100 \mathrm{rpm}$ and different vibration levels (triangle no vibration, diamond level 1, circle level 2, square level 3)

low agitation rates. Results also indicate that the sensitivity of the dissolution process of drug formulations toward operational parameters such as vibration varies considerably from product to product, that control of vibration is more important for some products than for others but definitely must be addressed when establishing dissolution specifications.

Based on this, it should be clear that critical vibration levels and vibration limits cannot be determined by simply screening vibration effects on dissolution of just one single drug formulation. Rather than this, an appropriate set of formulations which are supposed to be sensitive to the applied vibration would be required or vibration would need to be properly monitored in every single dissolution experiment. The set of formulations screened in the present series of experiments, however, can only present a starting point. In this first series of experiments, rapidly releasing formulations were examined. However, such formulations are often not as sensitive toward disturbances (e.g., vibrations) within a dissolution experiment as are formulations that dissolve over a longer time period (formulations that are poorly soluble, modified release formulations, etc.). For this reason, in a next step, formulations with longer overall dissolution times should also be screened at different vibration levels. Moreover, in future experiments, several other important questions would need to be addressed. These include the questions on how and at which location on different apparatus or in the dissolution lab representative vibration levels can or should be measured, what are typical vibration levels in a dissolution lab, how to potentially implement vibration measurements into the mechanical calibration procedure, and, finally, if provided that similar vibration levels are measured in different lab environments or on different dissolution apparatus for a given product similar dissolution profiles will be obtained when applying an official QC method.

\section{CONCLUSION}

Results of the present study show that the dissolution process of oral drug formulations can be affected by vibration. However, it also becomes clear that the degree to which a certain level of vibration impacts dissolution is strongly dependent on several factors such as drug properties, formulation parameters, and the design of the dissolution method. To ensure the establishment of robust and predictive dissolution test methods, the impact of variation should thus be considered in method design and validation. However, as it is obvious that until now the impact of vibration on dissolution results is not clearly understood, more studies will be required to better understand typical vibration levels that can arise in an analytical laboratory environment, how and where to really measure vibration during a dissolution experiment, how to standardize vibration measurement, and how to implement vibration screening into method validation and/or routine dissolution testing.

\section{ACKNOWLEDGMENTS}

The author would like to thank ERWEKA GmbH for providing vibration source, accelerometers, and software for the duration of the experiments and finally Dr. Zongming Gao, FDA, CDER for many interesting and helpful discussions. Part of this work has been presented at the 2012 AAPS Annual Meeting and Exposition, October 14-18, 2012, Chicago IL, USA.
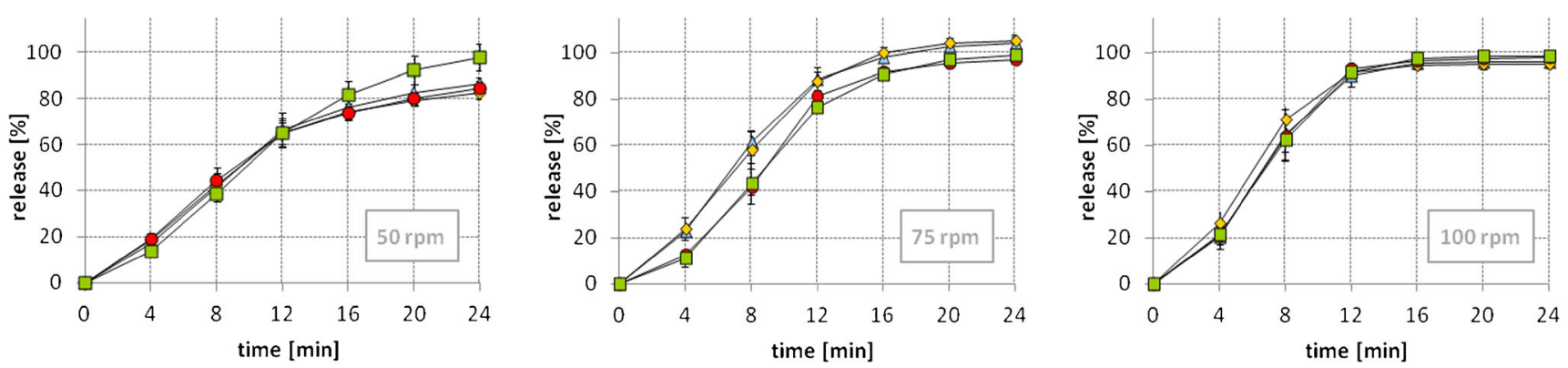

Fig. 6. Drug release (mean of $n=6 \pm \mathrm{SD}$ ) of Furosemide AL $40 \mathrm{mg}$ tablets at paddle speeds of 50, 75, and 100 rpm and different vibration levels (triangle no vibration, diamond level 1, circle level 2, square level 3) 


\section{REFERENCES}

1. Gray V, Kelly G, Xia M, Butler C, Thomas S, Mayock S. The science of USP 1 and 2 dissolution: present challenges and future relevance. Pharm Res. 2009;26(6):1289-302.

2. DeStefano AJ, Hauck WW, Stippler ES, Brown WE, Li C, Huang $\mathrm{GG}$, et al. Establishing new acceptance limits for dissolution performance verification of USPC apparatus 1 and 2 using USPC prednisone tablets reference standard. Pharm Res. 2011;28(3):505-16.

3. Kaniwa N, Katori N, Aoyagi N, Ishigame N, Kojima S. Collaborative study on the development of a standard for evaluation of vibration levels for dissolution apparatus. Int J Pharm. 1998;175:119-29.

4. Crist B, Spisak D. Evaluation of induced variance of physical parameters on the calibrated USP dissolution apparatus 1 and 2. Dissolut Technol. 2005;12(1):28-31.

5. Vangani S, Flick T, Tamayo G, Chiu R, Cauchon N. Vibration measurements on dissolution systems and effects on dissolution of prednisone Tablets RS. Dissolut Technol. 2007;14(1):6-14.

6. Burmicz J. Technical note: why is vibration an issue for dissolution testing? Dissolut Technol. 2008;15(1):29-31.

7. Martin GP, Gray VA. Overview of dissolution instrument qualification. Including common pitfalls. Dissolut Technol. 2011;18(2):6-10.
8. Beyer WF, Smith DL. Unexpected variable in the USP-NF rotating dissolution test. J Pharm Sci. 1971;60(3):496-7.

9. Embil K, Torosian G. Effect of instrumental vibration levels on dissolution. J Pharm Sci. 1979;68(10):1336-8.

10. Gao Z, Moore TW, Doub WH. Vibration effects on dissolution tests with USP apparatuses 1 and 2. J Pharm Sci. 2008;97(8):333543.

11. Gao Z, Moore TW, Buhse LF, Doub WH. The random vibration effects on dissolution testing with USP apparatus 2. J Pharm Sci. 2009;98(1):297-306.

12. Gao Z, Thies A, Doub W. Vibration effects of lab equipment on dissolution testing with USP paddle method. J Pharm Sci. 2010;99(1):403-12.

13. Amidon GL, Lennernas H, Shah VP, Crison JR. A theoretical basis for a biopharmaceutic drug classification: the correlation of in vitro drug product dissolution and in vivo bioavailability. Pharm Res. 1995;12(3):413-20.

14. Lindenberg M, Kopp S, Dressman JB. Classification of orally administered drugs on the World Health Organization Model list of Essential Medicines according to the biopharmaceutics classification system. Eur J Pharm Biopharm. 2004;58(2):265-78.

15. FDA. Guidance for industry: the use of mechanical calibration of dissolution apparatus 1 and 2 - Current good manufacturing practice (CGMP). Silver Spring: U.S. Department of Health and Human Services Food and Drug Administration Center for Drug Evaluation and Research (CDER); 2010. 